\title{
SOME STRUCTURAL PROPERTIES OF HAUSDORFF MATRICES
}

\author{
BY B. E. RHOADES \\ Communicated by Einar Hille, October 10, 1958
}

1. Definitions. Let $A=\left(a_{n k}\right)$ denote an infinite matrix. $A$ is called conservative if $A$ has finite norm, $a_{k}=\lim _{n \rightarrow \infty} a_{n k}$ exists for each $k$, and $\lim _{n \rightarrow \infty} \sum_{k} a_{n k}$ exists. $A$ is called multiplicative if $A$ is conservative and $a_{k}=0$ for each $k$.

$s$ denotes the space of sequences, $m$ the subspace of bounded sequences, and $c$ the subspace of convergent sequences. $E_{1}$ is the field of complex numbers and $E_{\infty}$ the set of sequences, each of which possesses only a finite number of nonzero terms.

Let $x$ be a fixed sequence. Then $c \oplus x=\{y+x \mid y \in c\}$.

Let $H=\left(h_{n k}\right)$ denote a Hausdorff matrix generated by a sequence $\mu$. I shall use $(H, \mu)$ to denote the convergence domain of $H, H_{\mu}$ to denote the matrix, and $H \sim \mu$ to denote the method.

A matrix $A=\left(a_{n k}\right)$ is said to be of property $\mathrm{P}$, displacement $m$ (written $c^{m} A$ is of property $\mathrm{P}$ ) if, for all $k \geqq m, a_{n k}$ possesses property P.

A corridor matrix is a matrix with the property that there exists a positive integer $r$ such that $a_{n k}=0$ for all $n$ and $k$ with $|n-k|>r$. The smallest such $r$ denotes the width of $A$.

2. Introduction. Let $\mathrm{H}$ denote the set of Hausdorff matrices with finite norm. $\mathrm{H}$ coincides with the set of conservative Hausdorff matrices as a result of [1, page 256 , lines 8-12].

Hille [2] denotes the set of all multiplicative Hausdorff matrices by $M$, and observes that it forms a commutative Banach algebra which is also an integral domain. Hence the concepts of unit, prime, divisibility, associate, multiple, and factor can be defined in $M$. Hille and Tamarkin $[3$, p. 576; 4, p. 907] observed that every moment function $\mu(z)$ of the form $\mu(z)=(z-a) /(z+b), Q(a)>0, Q(b)>0$, is prime in $M$; i.e., $H \sim \mu$ is not equivalent to convergence, but includes only methods that are equivalent to convergence. Hille mentioned this fact in [2, p. 422], and again raised the open question as to whether all primes in $M$ are of this form.

From Hille's definition of a prime moment function, a regular Hausdorff matrix $H$ with the property that $(H, \mu)=c \oplus x$ for some unbounded sequence $x$ would have a moment function $\mu(z)$ which would be a prime element of $M$. The results stated in this paper show that it is impossible to construct a Hausdorff matrix $H \in \mathrm{H}$ 
with $(H, \mu)=c \oplus x$, for some unbounded $x$, by the technique of Zeller [5].

3. Results. The proofs of the following theorems stem from the definition of a Hausdorff matrix, and use the fact that knowledge of the values of any two of the terms $h_{n, k}, h_{n+1, k}, h_{n+1, k+1}$ gives information about the third. For example, if for some $n$ and $k h_{n, k}$ $=h_{n+1, k}=0$, then $h_{n+1, k+1}=0$. See $[1$, p. 255, line 14].

Theorem 1. Suppose that for some integer $k,\left\{h_{n k}\right\} \in E_{\infty}$. Then $c^{k} H$ is a corridor matrix of width $N-k$.

Theorem 2. Let $h_{n k}=\alpha_{n}$ for $k=0,=0$ for $k>0, \alpha_{n} \in E_{1}$. Then $H$ is a Hausdorff matrix $\leftrightarrow \alpha_{n}=c, c$ a constant.

THeOREM 3. Let $H$ be triangular with $h_{n, 0}=c, c \in E_{1}$, for all $n$. Then $H$ is Hausdorff $\leftrightarrow h_{n, k}=0$ for all $k>0$.

THEOREM 4. There does not exist a Hausdorff corridor matrix of width $k>1$ with the elements on the kth diagonal all the same number $c$.

THEOREM 5. Let $H$ be a Hausdorff matrix with its $(k+1)$ th column an element of $E_{\infty}$. Then, for $k \neq 0$, either the kth column is an element of $E_{\infty}$ or it has no two succeeding elements the same number.

THEOREM 6. Suppose that $H$ is a Hausdorff corridor matrix of width $r>1$. Then $H \notin \mathrm{H}$. For $r=1, \mu_{0} \neq 0, H$ is a diagonal matrix and hence is equivalent to convergence.

THEOREM 7. Let $c^{m} H$ be a corridor matrix of width $r>1$. Then $H \notin \mathrm{H}$.

Theorem 8. If $c^{m} H$ is a diagonal matrix, $m>0$, then $\mu_{k}=\mu_{k+1}$ for $k \geqq m$.

TheOREM 9. Let $c^{m} H$ be a diagonal matrix, $m>0$. Then $c^{m} H$ cannot have $\left\{h_{n k}\right\} \in E_{\infty}$ for $0 \leqq k \leqq m-1$.

TheOREM 10. Let $c^{m} H$ be a diagonal matrix, $m>0$. Then $h_{n, m-1} \neq 0$ for $n>m-1$.

THEOREM 11. Let $c^{m} H$ be a null matrix, $m>0$; i.e., $h_{n k}=0$ for all $n \geqq k \geqq m$. Then $\mu_{m-1} \neq 0$.

Theorem 12. If $c^{m} H$ is a diagonal matrix, $m>1$, then $H \notin \mathrm{H}$.

4. Conclusion. Combining the results of the above set of theorems we see that $\mathrm{H}$ is composed of two types of matrices. For $H \in \mathrm{H}$, either $c^{m} H$ is a diagonal matrix for $m=0,1$, or $H$ has no column an element of $E_{\infty}$. 
Let $D=\left\{H_{\mu} \mid \mu=\left\{\mu_{0} c, c, \cdots\right\}, \mu_{0}, c \in E_{1}\right\}$. Then $D$ is a Banach algebra.

In constructing a regular matrix $A$ with $c_{A}=c \oplus x$ for some unbounded $x$, Zeller [5] uses a nondiagonal matrix with columns which are elements of $E_{\infty}$. However, such a construction is not possible for any $H \in \mathrm{H}$, hence, a fortiori, not possible for any regular $H$.

It is still, as far as I know, an open question whether there exists a Hausdorff matrix $H \in \mathrm{H}$ with $(H, \mu)=c \oplus x$ for some unbounded $x$.

\section{REFERENCES}

1. G. H. Hardy, Divergent series, Oxford, 1949.

2. E. Hille, Functional analysis and semi-groups, Amer. Math. Soc. Colloquium Publication, vol. 31, 1948.

3. E. Hille and J. D. Tamarkin, Questions of relative inclusion in the domain of Hausdorff means, Proc. Nat. Acad. Sci. U.S.A. vol. 19 (1933) pp. 573-577.

4. - On moment functions, Proc. Nat. Acad. Sci. U.S.A. vol. 19 (1933) pp. 902-908.

5. K. Zeller, Merkwïrdigkeiten bei Matrixverfahren; Einfolgenverfahren, Arch. Math. vol. 4 (1953) pp. 1-5.

Lafayette College 